\title{
Establishing Flask-Relevant Reaction Conditions for Imaging Bimetallic Nanocrystal Formation with Liquid Phase Transmission Electron Microscopy
}

Mei Wang ${ }^{1}$ and Taylor Woehl ${ }^{2}$

${ }^{1}$ University of Maryland, College Park, Maryland, United States, ${ }^{2}$ University of Maryland, Columbia, Maryland, United States

Liquid phase transmission electron microscopy (LP-TEM) has enabled unprecedented insights into the crystallization mechanisms of solution phase inorganic and organic nanoparticles. ${ }^{1-3}$ Most LP-TEM studies of nanoparticle formation utilize the electron beam as a stimulus for nanoparticle formation. Generally, a solution phase inorganic or organic metal precursor is reduced by radicals created by radiolysis of the solvent by the TEM beam. ${ }^{3}$ While LP-TEM has yielded many high impact studies on nanocrystal formation, the question remains whether the chemical reactions produced by the TEM beam, and the resulting kinetics and mechanisms of nanocrystal formation, are comparable to those occurring in a flask-based synthesis. This question must be addressed if LP-TEM experiments are expected to produce useful insights that will guide synthesis of nanocrystals.

There are several notable differences between electron beam induced nanocrystal synthesis during LPTEM and flask-based synthesis on the bench top. Flask-based synthesis occurs in a batch reactor, where reducing agent is rapidly injected at the beginning of synthesis, while the electron beam continuously injects reducing agent into the solution during LP-TEM synthesis. Reducing radicals that convert precursors to nanocrystals during LP-TEM synthesis, such as solvated electrons and hydrogen radicals, are highly reactive compared to chemical reducing agents. ${ }^{4}$ The immediate consequence of this difference is modified precursor reduction kinetics compared to chemically stimulated nanocrystal formation. Oxidizing radicals also form during LP-TEM, which readily react with polymers and small organic molecules that are commonly utilized as capping ligands during nanocrystal synthesis. These differences, among others, must be carefully considered during a LP-TEM experiments to interpret the nanocrystal formation mechanisms and glean practical insights about the corresponding flask-based synthesis.

To this end, we have undertaken a rigorous comparison of LP-TEM and flask-based methods for synthesis of bimetallic alloyed gold/copper $(\mathrm{AuCu})$ nanoparticles. Alloying gold and copper together is notoriously difficult because gold chloride precursor reduces more rapidly than copper ions. Millstone et al. have explored the use of bimetallic precursor-ligand complexes for promoting alloying and have shown these complexes enable efficient alloying of gold and copper. ${ }^{5}$ However, the alloying mechanism remains unclear. Flask-based synthesis of AuCu nanocrystals using sulfhydryl functionalized polyethylene glycol (SH-PEG) as a complexing and capping agent and sodium borohydride as a strong reducing agent formed single crystal $~ 2-3 \mathrm{~nm}$ diameter alloyed AuCu nanocrystals (Figure 1a,b). We modified the flask synthesis recipe by decreasing the precursor and SH-PEG concentrations by 50 times and formed nanocrystals using LP-TEM (Figure 1b-d). Time-lapsed LP-TEM images show the formation of 3-7 nm nanocrystals over a time scale of $\sim 30 \mathrm{~s}$. Nanocrystals were single crystal and non-aggregated when low magnification $(\mathrm{M}<$ $500 \mathrm{kX}$ ) and low beam current $(<150 \mathrm{pA}$ ) were utilized for electron beam induced synthesis (Figure 2a). However, at higher magnification and beam current, significant aggregation of the nanoparticles was observed, ostensibly due to degradation of the PEG-SH capping ligands by radicals (Figure $2 b$ ). We systematically varied the electron beam magnification and beam current and assessed the size distribution and aggregation of the resulting nanocrystal using ex situ HR-TEM. Figure 2c shows the particle size 
distributions for LP-TEM synthesis at different magnifications compared to flask based synthesis. Clearly the flask synthesized nanocrystals are smaller with a lower dispersity. Interestingly, increasing the magnification decreased the preference to form $\sim 3 \mathrm{~nm}$ diameter particles in favor of forming either aggregated particles or particles $<2 \mathrm{~nm}$. This is likely due to the increased nucleation rate at high magnification together with the increased concentration of oxidizing radicals that damage capping ligands. The degree of nanoparticle aggregation was assessed by quantifying the eccentricity of the nanocrystals, which is a quantitative measure of elliptical shape ( 0 is a perfect sphere, 1 is a line, values in between are ellipses) [6]. This comparative study enables identifying key differences between LP-TEM synthesis and flask-based synthesis of bimetallic nanocrystals towards establishing equivalent synthesis conditions between the two synthesis methods. ${ }^{6}$
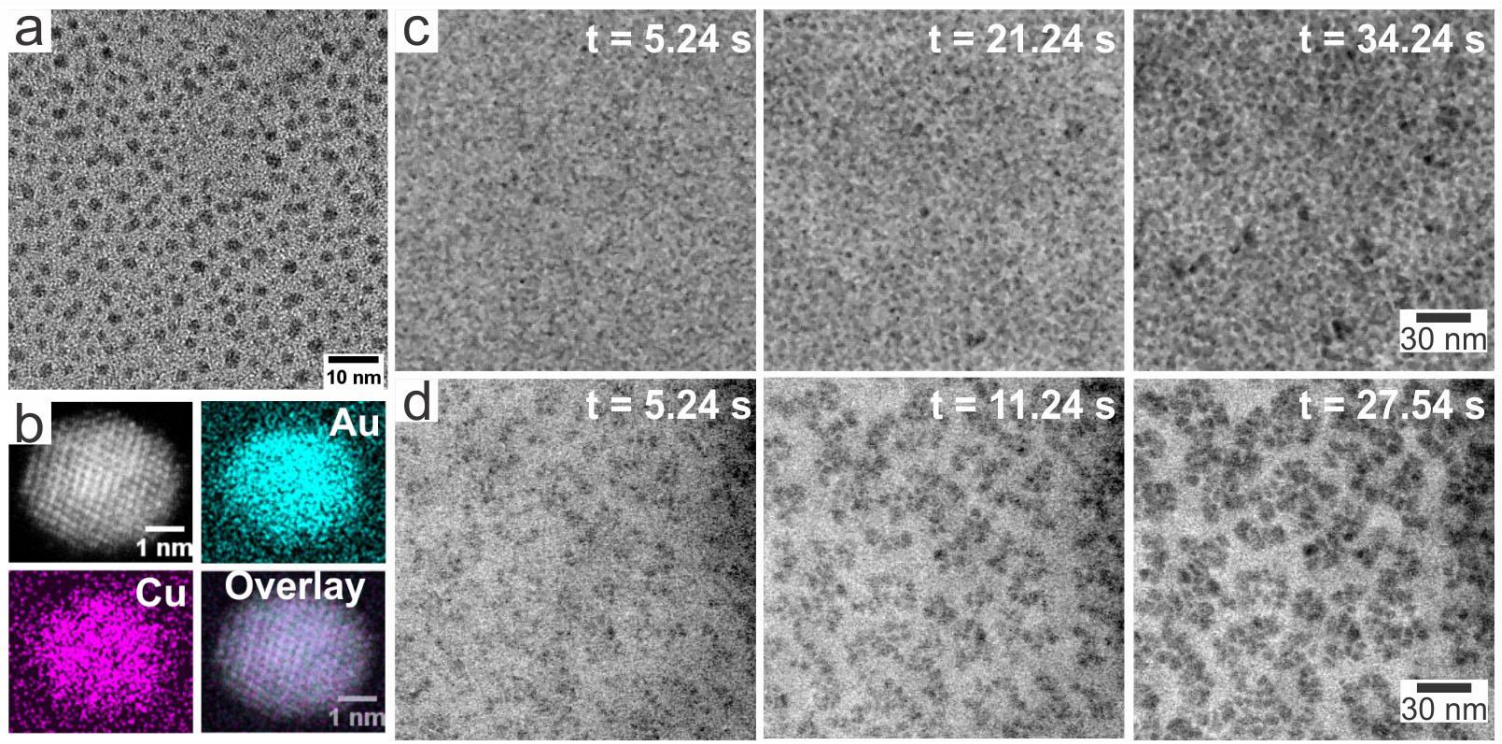

Figure 1. (a) HRTEM image of AuCu nanocrystals formed by flask synthesis. (b) HAADF-STEM image (top left) and EDS maps of copper and gold showing alloy formation in AuCu nanocrystals. (c) LP-TEM time lapsed series of images taken at $\mathrm{M}=500,000$ and beam current of $16 \mathrm{pA}$ (dose rate $=16.7 \mathrm{MGy} / \mathrm{s}$ ). (d) LP-TEM time lapsed series of images taken at $\mathrm{M}=500,000$ and beam current of $74 \mathrm{pA}$ (dose rate $=77.1 \mathrm{MGy} / \mathrm{s}$ ).
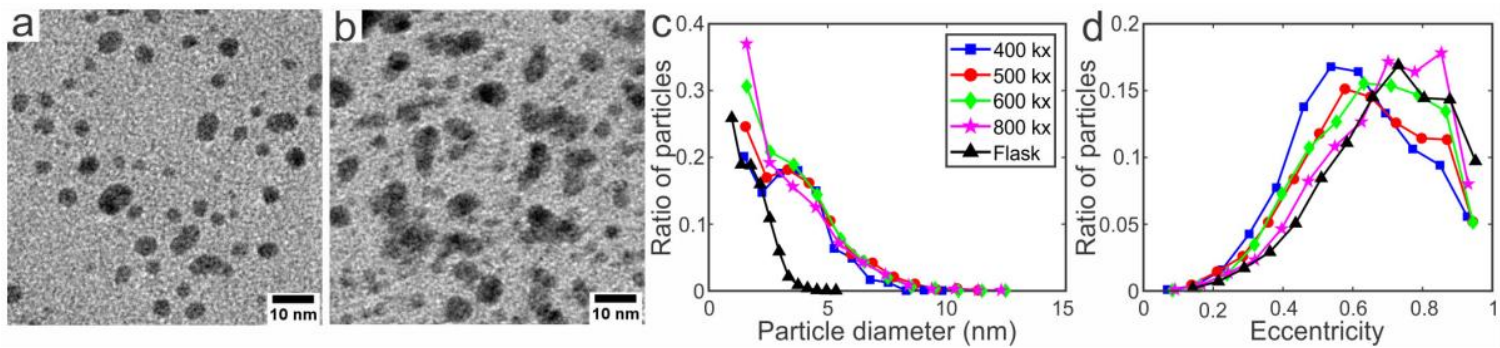

Figure 2. Ex situ HR-TEM images of AuCu nanocrystals formed by LP-TEM at (a) M = 400,000, beam current of $16 \mathrm{pA}$ (dose rate $=7.3 \mathrm{MGy} / \mathrm{s}$ ) and (b) $\mathrm{M}=600,000$, beam current of $141 \mathrm{pA}$ (dose rate $=210$ MGy/s). Particle size distributions (c) and particle eccentricity (d) for LP-TEM synthesis at different magnifications compared to flask based synthesis.

References

[1] Zheng, H. M., Smith, R. K., Jun, Y. W., et al., Science, 324 (2009), p. 1309-1312. 
[2] Patterson, J. P., Abellan, P., Denny, M. S., et al., J. Am. Chem. Soc., 137 (2015), p. 7322-7328.

[3] Woehl, T. J., Evans, J. E., Arslan, L., et al., Acs Nano, 6 (2012), p. 8599-8610.

[4] Woehl, T. J., Abellan, P., J. Microsc., 265 (2017), p. 135-147.

[5] Marbella, L. E., Chevrier, D. M., Tancini, P. D., J. Am. Chem. Soc., 137 (2015), p. 15852-15858.

[6] We acknowledge funding support from ACS PRF (\#61111-DNI10). 